\title{
Prediction of Extubation Readiness Using Transthoracic Ultrasound in Preterm Infants
}

\author{
Reem Soliman ${ }^{1}$, Yasser Elsayed ${ }^{2}$, Reem Said ${ }^{1}$, Abdulaziz Abdulbaqi ${ }^{1}$, Rania Hashem ${ }^{1}$, and \\ Hany $\mathrm{Aly}^{3}$ \\ ${ }^{1}$ Cairo University Kasr Alainy Faculty of Medicine \\ ${ }^{2}$ University of Manitoba College of Medicine \\ ${ }^{3}$ Cleveland Clinic Children's Hospital
}

January 7, 2021

\begin{abstract}
We aimed to test the hypothesis that a lung ultrasound severity score (LUS) and assessment of left ventricular eccentricity index of the interventricular septum (LVEI) by focused heart ultrasound can predict extubation success in mechanically ventilated infants. We conducted a prospective study on premature infants $<34$ weeks' of gestation. LUS was performed on postnatal days 3 and 7 by an investigator who was masked to infants' ventilator parameters. LVEI and pulmonary artery pressure (PAP) were measured at postnatal day 3. A receiver operator curve was constructed to assess the ability to predict extubation success. Spearman correlation was performed between LVEI and PAP. A total of 104 studies were performed to 66 infants; of them 39 had mild and 65 had moderate-severe lung disease. LUS predicted extubation success with a sensitivity and a specificity of $91 \%$ and $69 \%$, respectively. Area under the curve was 0.83 (CI: 0.75-0.91). LVEI did not differ between infants that succeeded and failed extubation. It correlated with PAP during systole $(\mathrm{r}=0.66)$. We conclude that LUS predicts extubation success in mechanically ventilated preterm infants whereas LVEI correlates with high PAP.
\end{abstract}

\section{Introduction}

Respiratory distress syndrome (RDS) is the most common cause of neonatal morbidity and mortality in preterm infants ${ }^{1}$. The incidence of RDS is inversely proportional to gestational age, occurring in $>90 \%$ in preterm infants born $<28$ weeks ${ }^{2}$. These infants are rescued with surfactant administration via an endotracheal tube and supported with nasal continuous positive airway pressure after a brief period of mechanical ventilation. The European guidelines for management of RDS in preterm infants reserve the use of mechanical ventilation to rescue babies who are not responding to positive pressure ventilation via face mask nasal prongs. Tracheal extubation is expected to ensue shortly after surfactant administration and stabilization of oxygenation $^{3}$. However, due to certain limitations, this practice has not been fully adopted outside the Western world, thereby allowing mechanical ventilation for several days.

Both mechanical ventilation and tracheal intubation are independently associated with intraventricular hemorrhage in premature infants ${ }^{4}$. The exposure of infants to multiple intubations is not a safe practice. Studies demonstrated significant hemodynamic derangements that occur during intubation of premature infants ${ }^{5}$. Consequently, intubation has been associated with increased risk for intraventricular hemorrhage in premature infants ${ }^{4}$. Since there is no clear criteria to guide clinicians when to extubate, infants may not necessarily succeed the extubation attempt and are subsequently re-intubated. Therefore, there is an unmet need to device an indicator for readiness to extubate in order to avoid the risks associated with re-intubation.

Lung ultrasound can recognize a normal aerated lung in contrast to interstitial or alveolar patterns. In the last decade, lung ultrasounds have been increasingly used in critically ill patients, and evidence based 
international guidelines are published for the use of lung ultrasounds in adult critical care ${ }^{6}$. It is simple and raises no threat of radiation. Evidence-based guidelines for lung ultrasound utilization in neonates have been recently published ${ }^{7}$.

Echocardiography is considered the gold standard tool to detect anatomical cardiovascular defects, assess cardiac function, evaluate abnormal pulmonary circulation and estimate the response to therapeutic interventions. However, it requires specific skills and detailed training for a caregiver to perform neonatal echocardiography ${ }^{8}$. Focused heart ultrasound is a simplified protocol of bedside ultrasound screening of pulmonary hypertension by measuring left ventricular eccentricity index (LVEI). LVEI is a quantifiable measure of the amount of distortion of ventricular septal geometry that is related to increased right ventricular systolic or diastolic pressures and volumes. LVEI has been associated with pulmonary hypertension in children and adults but has not been validated in premature infants ${ }^{9}$.

This prospective study was conducted on premature infants supported with mechanical ventilation for several days. Infants had point of care lung ultrasound and LVEI measurements. The aim of this study was to test the hypothesis that a LUS combined with LVEI would predict success of extubation in mechanically ventilated preterm infants. In addition, correlations of LVEI with pulmonary artery pressures and patent ductus arteriosus were made.

\section{Materials and Methods}

\section{Patients :}

This prospective cohort study was conducted at the neonatal intensive care unit of Kasr El Aini Hospital, a referral tertiary care university hospital. The study was approved by the Institutional Research Ethics Board and informed parental consents were obtained for all subjects before recruitment. Infants were included in the study if they fulfilled the following criteria: a) gestational age $<34$ weeks, b) postnatal age $<48$ hours, and c) supported with invasive mechanical ventilation for initial presentation with RDS. Infants were excluded if they were extubated before 3 days of life or if they had any of the following: a) major congenital anomalies, b) perinatal asphyxia, or c) hemodynamic instability managed by inotropic administration at the time of screening.

Demographic and clinical data were collected from the chart. Those data included the use of antenatal steroids, infant's sex, gestational age (GA), birth weight (BW), small for gestational age status, Apgar scores at 5 and 10 minutes, perinatal confirmed infections, and intraventricular hemorrhage. Clinical parameters of hemodynamic stability including systolic and diastolic blood pressures, heart rate, urine output, and lactic acid at the time of assessment were collected. Data on respiratory outcomes were collected; bronchopulmonary dysplasia (BPD) was defined by the use of respiratory support or supplemental oxygen to maintain oxygen saturation $>90$ by pulse oximeter at 36 weeks of postmenstrual age for infants born [?] 32 weeks of gestation and the use of supplemental $\mathrm{O}_{2}$ at 28 days for infants born at 33-34 weeks of gestation ${ }^{10}$.

LUS and transthoracic focused cardiac ultrasound (FCUS) were performed on postnatal days 3 and 7 for all recruited infants. The radiologist who performed the studies (R.H.) was masked to the clinical details of infants; likewise, the managing team was blinded to the ultrasound results. If an infant was extubated after 3 days and before 7 days, he/she would have only one study at 3 days of life.

\section{Clinical respiratory parameters:}

Respiratory data were collected for all infants during the first 7 days of life. Data included mean airway pressure (MAP), fraction of inspired oxygen $\left(\mathrm{FiO}_{2}\right)$, arterial blood gas measurements, total mechanical ventilation days, total oxygen days, and success/failure of extubation events. Oxygen saturation index (OSI) was calculated on postnatal days 3 and 7 ; OSI $=\mathrm{MAP} \times \mathrm{FiO}_{2} \mathrm{x} 100 /$ arterial oxygen saturation ${ }^{10}$. We stratified respiratory severity into mild, moderate, and severe based on OSI of [?] 2.9, 3.0-6.4, and [?]6.5, respectively ${ }^{11}$.

\section{Extubation criteria:}


Respiratory management in this center primarily used CPAP to support premature infants with GA $>28$ weeks, otherwise infants were routinely intubated. Intubated infants were supported with mechanical ventilation for several days. Weaning off mechanical ventilation to extubation followed standardized guidelines. Gradual weaning of $\mathrm{FIO}_{2}$ and peek inspiratory pressure (PIP) or tidal volume (VT) were performed guided by assessment of chest excursion, oxygen saturation and blood gases results. Infants were extubated when ventilatory rate was $<25$ breaths/min and PIP at $16-18 \mathrm{cmH}_{2} \mathrm{O}$ that delivered desired VT. The process of weaning would typically require several days. The weaning practice did not have any additional "readiness test" before extubation. Infants were extubated to nasal CPAP or nasal non-invasive ventilation based on the observed work of breathing and presence of irregular breathing or apnea.

\section{Lung ultrasound protocol ${ }^{12}$ :}

A total of six lung zones were scanned, 3 on the right lung and 3 on the left lung [13]. These zones are summarized in Figure 1. Four patterns of lung findings were described (Figure 2) with graded severity score; the lowest score is zero and the highest is 3 for each zone (Supplementary Figures S1-S4). Therefore, the total lung score is 18 , which is the sum of the 3 lung zones on both right and left sides $(3 \times 3 \times 2=18)^{12}$.

\section{Focused cardiac ultrasound ${ }^{9}$ :}

FCUS was done on postnatal days 3 and 7 by the same radiologist (R.H.) who was experienced with neonates and was blinded to clinical details of the studied infants. LVEI was measured at end-systole from the parasternal short axis 2D image at the mid-papillary muscle level. The formula (LVEI = D2/ Dl) was used where: $\mathrm{Dl}$ is the ventricular diameter perpendicular to the interventricular septum bisecting D2, and D2 is the diameter parallel to the interventricular septum. LVEI was considered abnormal when the ratio was $>1.0$ and a higher LVEI was considered a marker of increased pulmonary vascular resistance ${ }^{13-15}$. In addition, full echocardiography was done by pediatric cardiologist to screen for congenital heart disease and patent ductus arteriosus (PDA) and to measure PAP assessment on day 3 of life. Both lung and cardiac ultrasounds were performed using the Toshiba Nemio 30 Ultrasound System with the Toshiba PLM-805AT linear array ultrasound transducer probe at a frequency of $5-10 \mathrm{MHz}$ (Toshiba, Tokyo, Japan) gray scale B mode examination.

PDA was visualized using 2D echocardiography and confirmed by color Doppler, PDA was considered hemodynamically significant if the left atrium to aortic root ratio in motion mode echocardiography exceeded 1.4 and if PDA size exceeded $1.5 \mathrm{~mm}{ }^{16}$. The pulmonary systolic pressure was determined by calculating the systolic pressure gradient peak between the right ventricle and the right atrium assessed through the simplified Bernoulli 7 equation $\left(4 \times \mathrm{V}^{2}\right)$, where $\mathrm{V}=$ peak systolic velocity of tricuspid regurgitation (TR) measured using continuous Doppler. The right atrial pressure was measured according to the collapse of the inferior venous cava during inspiration. The right ventricle systolic pressure, in absence of right ventricle outlet obstruction, is similar to the pulmonary systolic pressure. An estimated systolic pulmonary artery pressure exceeding $35 \mathrm{mmHg}$ was considered pulmonary hypertension in preterm neonates ${ }^{17}$.

\section{Statistical and power analysis:}

SPSS version 25 (SPSS Inc., Chicago, IL) was used to perform the statistical analysis. Data were presented as median with interquartile range or frequencies. Comparison between groups was analyzed by Mann-Whitney $\mathrm{U}$ test. Frequencies were analyzed by chi-square test; $\mathrm{p}$ values $<0.05$ were considered significant. Receiver operator curve (ROC) was constructed and predictive values were calculated. Spearman's correlation was used to examine correlations of LVEI with PAP.

Power analysis was done comparing extubation trial results. Chi squared test for independent samples was chosen to perform the power analysis, fixing the $\alpha$-error level at 0.05. Sample size was entered as 100 with success/failure ratio of $67 \%$ and $33 \%$. Accordingly, the calculated power was reported to be $>90 \%$.

\section{Results}

A total of 104 point of care US were performed to 66 infants at a median (IQR) GA 30 (29-31) weeks. Infants 
were classified into two groups based on the outcome of extubation trials; succeeded vs failed extubation. The two groups were comparable in demographic and clinical characteristics (Table 1). LUS differed significantly between the two groups $(\mathrm{p}=0.022)$ whereas LVEI did not differ between groups (Table 1 ).

Table 2 shows the respiratory characteristics of both groups; mean airway pressure, duration of mechanical ventilation and of oxygen therapy were significantly higher in group-2 compared to group-1. Other ventilator parameters were comparable between the two groups.

OSI was different between those who succeeded and failed extubation: 2.1 (1.5 - 2.6) vs 3.4 (2.7-4.7), respectively; $\mathrm{p}<0.0001$. Duration of mechanical ventilation was significantly increased in infants who failed extubation: $5(5-8)$ days vs $8.5(8-9)$ days, $\mathrm{p}<0.0001$. Comparisons of the study population based on LUS severity are presented in supplemental tables (Tables S1 and S2).

Multivariate logistic regression was performed with extubation success as the dependent outcome and LUS, LVEI and duration of mechanical ventilation as covariates. In this model, LVEI remained insignificant ( $\mathrm{p}=0$. $817)$.

LUS was able to predict extubation success at day3 (area under the curve $0.71,95 \%$ CI: $0.55-0.88, \mathrm{p}=0.023$ ) and at day 7 (area under the curve $=0.92,95 \%$ CI: $0.82-1.0, \mathrm{p}<0.001$ ) (Figure 3 ). Table 3 presents predictive values for extubation success with LUS cut off points at the highest sensitivity and specificity on days 3 and 7. LVEI did not predict extubation success in ROC analysis (area under the curve $=0.52,95 \%$ CI: $0.32-$ $0.71, \mathrm{p}=0.87$ ) (Figure 3). The predictive values for extubation success with LEVI are presented in Table 3. Using DeLong test, the predictive values for LUS and LVEI were compared and was found insignificant $(\mathrm{p}=0.19)$.

LVEI, measured at end-systole, correlated with PAP ( $\mathrm{r}=0.66,95 \%$ CI: $0.50-0.78, \mathrm{p}<0.001)$ and with internal diameter of the ductus arteriosus ( $\mathrm{r}=0.25,95 \%$ CI: 0.01-0.46, $\mathrm{p}=0.04$ ). (Supplement Figures S1-S4). A total of 32 infants had PPHN with PAP $>35 \mathrm{mmHg}$. There was no difference in PAP among infants who succeeded and failed extubation $(\mathrm{p}=0.27)$, Table 1 .

\section{Discussion}

This study demonstrated the ability of point of care LUS to predict success of extubation in mechanically ventilated premature infants. Infants with higher LUS were supported with higher venitlatory pressure. LVEI did not differ among infants who succeeded or failed extubation. However, it correlated with PAP.

Infants with higher LUS were supported with significantly higher pressure of conventional mechanical ventilation. The use of higher PIP is indicative of decreased compliance in infants with severe lung disease. A previous study of CPAP-supported infants demonstrated the need for higher pressure in infants with higher LUS $^{12}$. Oxygen saturation index (OSI) was also increased in infants with severe lung disease who had higher LUS. This finding agreed with previous studies that showed correlation of LUS with OSI and with the ratio of arterial oxygen saturation to $\mathrm{FiO}_{2}{ }^{12,15}$.

This study is the first to use LUS to predict success of extubation from mechanical ventilation in premature infants. Many lung ultrasound scores have been developed to assess lung aeration and guide respiratory care in adults especially those with restrictive lung disorders; LUS in this population is strongly recommended (level of evidence A) ${ }^{18}$. Compared to adult literature, lung ultrasound has not been adequately addressed in neonates although it is easier owing to the small patients' size and the absence of obesity or heavy musculature ${ }^{19}$. A few studies described the ultrasound patterns in common respiratory conditions in neonates, such as meconium aspiration syndrome, hyaline membrane disease, transient tachypnea of the neonate, and pneumothorax ${ }^{21-23}$. Other studies described the usefulness of LUS in predicting the need for invasive mechanical ventilation in infants supported with noninvasive ventilation ${ }^{24,25}$ and the need for surfactant replacement in CPAP-supported extremely preterm neonates ${ }^{26}$. However, there is no study to predict success of extubation from mechanical ventilation. Giving the ease of its use at the bedside and the non-invasive nature of LUS, it is helpful to obtain LUS before extubation to avoid the exposure of infants to re-intubation should the extubation attempt fail. One of the main barriers to the more extensive use 
of the ultrasound technology in premature infants is the lack of efficient training solutions and the need to have structured quality-check assurance ${ }^{27}$. Once training is established the use of LUS can be the first-line imaging technique in preterm infants ${ }^{28}$.

Evaluating an infant for extubation readiness is clinically a challenge. There are significant variations in the decision making process of extubation; that lacks objective evidence-based criteria and is often contingent on caregiver experience. A recent international survey showed extubation readiness subject to caregiver personal interpretations of blood gas parameters and overall feeling of clinical stability of infants ${ }^{29}$. Some physicians advocate conducting apnea test before extubation, although this test is often conducted variably ${ }^{30}$. Therefore, it is important to have an objective method and/or a scoring system with a calculated prediction of success rate accordingly. LUS fulfils this unmet gap in neonatal practice. In this study, when LUS was [?]11extubation was successful in $>90 \%$ of infants.

Pulmonary hypertension in newborns (PPHN) is triggered by multiple etiologies including hypoxemia and underlying parenchymal lung diseases and can potentially hinder extubation ${ }^{28}$. Performing a full echocardiographic studies on all mechanically ventilated preterm infants would be exhaustive and may not consistently correlate with $\mathrm{PPHN}^{14}$. LVEI is a quantifiable measure of the amount of distortion of ventricular septal geometry due to elevated right ventricular systolic or diastolic pressures and/or volumes. Greater degrees of LVEI have been associated with PPHN in children and adults, but have not been studied thoroughly in premature infants. LVEI has the added benefit of being easily measured from any short axis view of the mid-left ventricle ${ }^{13}$. Increased end-diastolic LVEI would indicate volume overload as in hemodynamically significant PDA and increased end-systolic LVEI may indicate right ventricular volume overload as in PPHN 31. In the current study, systolic LVEI correlated with PAP that was measured concomitantly by echocardiography. It correlated with the presence of PDA as well. However, end-systolic LVEI did not correlate with OSI and did not prove to be valuable in predicting readiness for extubation. Of note, in this study we used LVEI only as a surrogate for pulmonary hypertension although a full assessment of PAP may have shown benefit in predicting extubation failure.

This study has the strength of addressing the success of extubation trials that is a real challenge in premature infants. The study has some limitations including the lack of comparison of LUS with findings of MRI or CT scan of the chest. To ensure consistency and reliability of the scores, all LUS studies were performed by the same investigator who was an experienced pediatric radiologist. However, these studies were well within the scope of POCUS and as suggested by international guidelines would be practically performed by neonatal caregivers with minimal experience with US ${ }^{7,32}$. Similarly, we have identified the US machine and proble models that were used for this research, although other devices with reasonable resolution will suffice $^{32}$. LUS addresses failure of extubation related to lung parenchymal disease. Other factors involved in extubation failure such as airway edema and apnea of prematurity cannot be predicted with LUS. Respiratory management in this center allows mechanical ventilation for several days, therefore the investigators advise using caution when extrapolating the current findings in settings that have different respiratory practices. In fact, being ventilated for several days, the respiratory failure for these enrolled infants would be better defined as an early stage of chronic pulmonary insufficiency rather than the classical respiratory distress syndrome ${ }^{33}$.

\section{Conclusions}

This study demonstrated the ability of LUS to predict success of extubation in premature infants with an AUC $>0.80$. The use of this simple bedside non-invasive test can potentially avoid the exposure of premature infants to multiple extubation- reintubation cycles.

Conflict of Interest: All authors declare no financial or conflict of interest in relation to this Funding: There is no funding source.

\section{References}


1. Kawakita T, Bowers K, Hazrati S, Zhang C, Grewal J, Chen Z, Sun L, Grantz KL. Increased neonatal respiratory morbidity associated with gestational and pregestational diabetes: a retrospective study. Am J Perinatol 2017;34(11):1160-1168. doi: 10.1055/s-0037-1604414

2. Spillane NT, Zamudio S, Alvarez-Perez J, Andrews T, Nyirenda T, Alvarez M, Al-Khan A. Increased incidence of respiratory distress syndrome in neonates of mothers with abnormally invasive placentation. PLoS One 2018;13(7),e0201266. doi: 10.1371/journal.pone.0201266.

3. Sweet DG, Carnielli V, Greisen G, Hallman M, Ozek E, te Pas A, Plavka R, Roehr CC, Saugstad OD, Simeoni U, Speer CP, Vento M, Visser GHA, Halliday HL. European consensus guidelines on the management of respiratory distress syndrome - 2019 update. Neonatology 2019;115(4):432-450. DOI: $10.1159 / 000499361$

4. Aly H, Hammad TA, Essers J, Wung JT. Is mechanical ventilation associated with intraventricular hemorrhage in preterm infants? Brain Dev 2012;34(3):201-205. doi: 10.1016/j.braindev.

5. Vedrenne-Cloquet M, Breinig S, Dechartres A, Jung C, Renolleau S, Marchand-Martin L, Durrmeyer $\mathrm{X}$. Cerebral oxygenation during neonatal intubation-ancillary study of the Prettineo-Study. Front Pediatr 2019;7:40. doi: 10.3389/fped.2019.00040

6. Volpicelli G, Elbarbary M, Blaivas M, Lichtenstein DA, Mathis G, Kirkpatrick AW, Melniker L, Gargani L, Noble VE, Via G, et al. International evidence-based recommendations for point-of-care lung ultrasound. Intensive Care Med 2012;38(4):577-591. doi: 10.1007/s00134-012-2513-4.

7. Singh Y, Tissot C, Fraga MV, Yousef N, Cortes RG, Lopez J, Sanchez-de-Toledo J, Brierley J, Colunga JM, Raffaj D et al. International evidence-based guidelines on Point of Care Ultrasound (POCUS) for critically ill neonates and children issued by the POCUS Working Group of the European Society of Paediatric and Neonatal Intensive Care (ESPNIC). Crit Care 2020;24(1):65. doi: 10.1186/s13054-0202787-9.

8. Elsayed YN, Fraser D. Integrated evaluation of neonatal hemodynamics, Part 2: Systematic bedside assessment. Neonatal Netw 2016;35(4):192-203. doi: 10.1891/0730-0832.35.4.192.

9. Elsayed Y, Abdelmawla M, Narvey M, Wrogemann J. A model of integrated lung and focused heart ultrasound as a new screening examination in infants at risk of respiratory or hemodynamic compromise. J Pediatr Neonatal Individ Med 2017;6(1):e060131. DOI: 10.7363/060131

10. Jobe AH, Bancalari E. Bronchopulmonary dysplasia. Am J Respir Crit Care Med 2001;163(7):17231729. doi: 10.1164/ajrccm.163.7.2011060.

11. Rawat M, Chandrasekharan PK, Williams A, Gugino S, Koenigsknecht C, Swartz D, Ma CX, Mathew B, Nair J, Lakshminrusimha S. Oxygen saturation index and severity of hypoxic respiratory failure. Neonatology 2015;107(3):161-166.

12. Brat R, Yousef N, Klifa R, Reynaud S, Shankar Aguilera S, De Luca D. Lung ultrasonography score to evaluate oxygenation and surfactant need in neonates treated with continuous positive airway pressure. JAMA Pediatr 2015;169(8):e151797 doi: 10.1001/jamapediatrics.2015.1797.

13. McCrary AW, Malowitz JR, Hornick CP, Hill KD, Cotten CM, Tatum GH, Barker PC. Differences in eccentricity index and systolic-diastolic ratio in extremely low-birth-weight infants with bronchopulmonary dysplasia at risk of pulmonary hypertension. Am J Perinatol 2016;33(1):57-62. doi: 10.1055/s0035-1556757.

14. Aggarwal S, Stockmann P, Klein MD, Natarajan G. Echocardiographic measures of ventricular function and pulmonary artery size: prognostic markers of congenital diaphragmatic hernia? J Perinatol 2011;31(8):561-566. doi: 10.1038/jp.2011.3.

15. Perri A, Riccardi R, Iannotta R, Di Molfetta DV, Arena R, Vento G, Zecca E. Lung ultrasonography score versus chest $\mathrm{X}$-ray score to predict surfactant administration in newborns with respiratory distress syndrome. Pediatr Pulmonol 2018;53(9):1231-1236. doi: 10.1002/ppul.24076.

16. Shepherd JL, Noori S. What is a hemodynamically significant PDA in preterm infants? Congint Heart Dis 2019;14(6):21-26. doi.org/10.1111/chd.12727

17. Parasuraman S, Walker S, Loudon BL, Gollop ND, Wilson AM, Lowery C, Frenneaux MP. Assessment of pulmonary artery pressure by echocardiography-A comprehensive review. Int J Cardiol Heart Vasc 2016;12:45-51. doi.org/10.1016/j.ijcha.2016.05.011 
18. Haddam M, Zieleskiewicz L, Perbet S, Baldovini A, Guervilly C, Arbelot C, Noel A, Vigne C, Hammad E, Antonini F, et al. Lehingue S. Lung ultrasonography for assessment of oxygenation response to prone position ventilation in ARDS. Intensive Care Med 2016;42(10):1546-1556. doi: 10.1007/s00134-0164411-7.

19. Raimondi F, Cattarossi L, Copetti R. International perspectives: Point-of-care chest ultrasound in the neonatal intensive care unit: An Italian perspective. NeoReviews 2014;15(1)e2-e6. DOI: https://doi.org/10.1542/neo.15-1-e2.

20. Piastra M, Yousef N, Brat R, Manzoni P, Mokhtari M, De Luca D. Lung ultrasound findings in meconium aspiration syndrome. Early Hum Dev 2014;90 (suppl 2):S41-S43. doi: 10.1016/S03783782(14)50011-4.

21. Copetti R, Cattarossi L, Macagno F, Violino M, Furlan R. Lung ultrasound in respiratory distress syndrome: a useful tool for early diagnosis. Neonatology 2008;94(1):52-59. doi: 10.1159/000113059.

22. Copetti R, Cattarossi L. The "double lung point": an ultrasound sign diagnostic of transient tachypnea of the newborn. Neonatology 2007;91(3): 203-209. doi.org/10.1159/000097454

23. Migliaro F, Sodano A, Capasso L, Raimondi F. Lung ultrasound-guided emergency pneumothorax needle aspiration in a very preterm infant. BMJ Case Rep 2014;pii: bcr2014206803. doi: 10.1136/bcr2014-206803.

24. Raimondi F, Migliaro F, Sodano A, Umbaldo A, Romano A, Vallone G, Capasso L. Can neonatal lung ultrasound monitor fluid clearance and predict the need of respiratory support? Crit Care 2012;16(6):R220. doi: 10.1186/cc11865.

25. Raimondi F, Migliaro F, Sodano A, Ferrara T, Lama S, Vallone G, Capasso L. Use of neonatal chest ultrasound to predict noninvasive ventilation failure. Pediatrics 2014;134(4): e1089-e10:94. doi:10.1542/peds.2013-3924

26. De Martino L, Yousef N, Ben-Ammar R, Raimondi F, Shankar-Aguilera S, De Luca D. Lung ultrasound score predicts surfactant need in extremely preterm neonates. Pediatrics 2018;142(3). pii: e20180463. doi: 10.1542/peds.2018-0463.

27. Kurepa D, Zaghloul N, Watkins L, Liu J. Neonatal lung ultrasound exam guidelines. J Perinatol 2018;38(1):11-22. doi: 10.1038/jp.2017.140.

28. Nair J, Lakshminrusimha S. Update on PPHN: mechanisms and treatment. Semin Perinatol 2014;38(2),78-91. doi: 10.1053/j.semperi.2013.11.004.

29. Shalish W, Sant' Anna GM.Respiratory care protocols in neonatal intensive care In: Aly H, AbdelHady H, editors. Respiratory management of newborns. London: InTechOpen; 2016.p 51-65.

30. Al-Mandari H, Shalish W, Dempsey E, Keszler M, Davis PG, Sant'Anna G. International survey on periextubation practices in extremely preterm infants. Arch Dis Child Fetal Neonatal Ed 2015;100(5):F428-F431. doi: 10.1136/archdischild-2015-308549.

31. Abraham S, Weismann CG. Left ventricular end-systolic eccentricity index for assessment of pulmonary hypertension in infants. Echocardiography 2016;33(6),910-915. doi: 10.1111/echo.13171.

32. Gomond-Le Goff C, Vivalda L, Foligno S, Loi B, Yousef N, De Luca D. Effect of different probes and expertise on the interpretation reliability of point-of-care lung ultrasound. Chest 2020;157(4),924-931.

33. Steinhorn R, Davis JM, Göpel W, Jobe A, Abman S, Laughon M, Bancalari E, Aschner J, Ballard R, Greenough A, Storari L, Thomson M, Ariagno RL, Fabbri L, Turner MA, International Neonatal Consortium. Chronic pulmonary insufficiency of prematurity: Developing optimal endpoints for drug development. J Pediatr 2017;191:15-21.e1. doi: 10.1016/j.jpeds.2017.08.006.

\section{Figure Legends}

Fig. 1 Lung zones used during ultrasound scanning [13]

R1: right upper anterior, R2: right lower anterior, R3: right lateral, same for the left side. The direction of the arrows represents the direction of the ultrasound probe.

Fig. 2 Four typical patterns of neonatal lung ultrasound

1) Normal lung areation with transverse repetition of pleural image which is A-line artifact (arrow), these 
patterns is given a severity score of zero. The absence of sliding in this study indicates the presence of a pneumothorax. 2) Longitudinal separated B lines (arrows) reaching the bottom of the image signifies interstitial fluid as in transient tachypnea of the newborn (TTN pattern). This pattern is given a severity score of 1. 3) Coalescent B lines with homogenous echographic view and thickened pleura as in respiratory distress syndrome (RDS pattern). This pattern is given a severity score of 2. 4) Subpleural air bronchogram (arrow) is seen in addition to the RDS pattern. This pattern is given a severity score of 3 (the highest score).

Fig. 3 Prediction of extubation success by lung ultrasound score and left ventricular eccentricity index

Receiver operating characteristic (ROC) curves for lung ultrasound scores at day 3 (Panel A), and day 7 (Panel B) and for left ventricular eccentricity index (Panel C). Area under the curve, $95 \%$ confidence interval, and $\mathrm{p}$ values are $(0.71,95 \% \mathrm{CI}: 0.55-0.88, \mathrm{p}=0.023),(0.92,95 \% \mathrm{CI}: 0.82-1.0, \mathrm{p}<0.001)$ and $(0.52,95 \%$ CI: $0.32-0.71, \mathrm{p}=0.87)$, respectively.

\section{Hosted file}

Table 1.pdf available at https://authorea.com/users/387979/articles/502849-prediction-ofextubation-readiness-using-transthoracic-ultrasound-in-preterm-infants

\section{Hosted file}

Table 2.pdf available at https://authorea.com/users/387979/articles/502849-prediction-ofextubation-readiness-using-transthoracic-ultrasound-in-preterm-infants

\section{Hosted file}

Table 3.pdf available at https://authorea.com/users/387979/articles/502849-prediction-ofextubation-readiness-using-transthoracic-ultrasound-in-preterm-infants

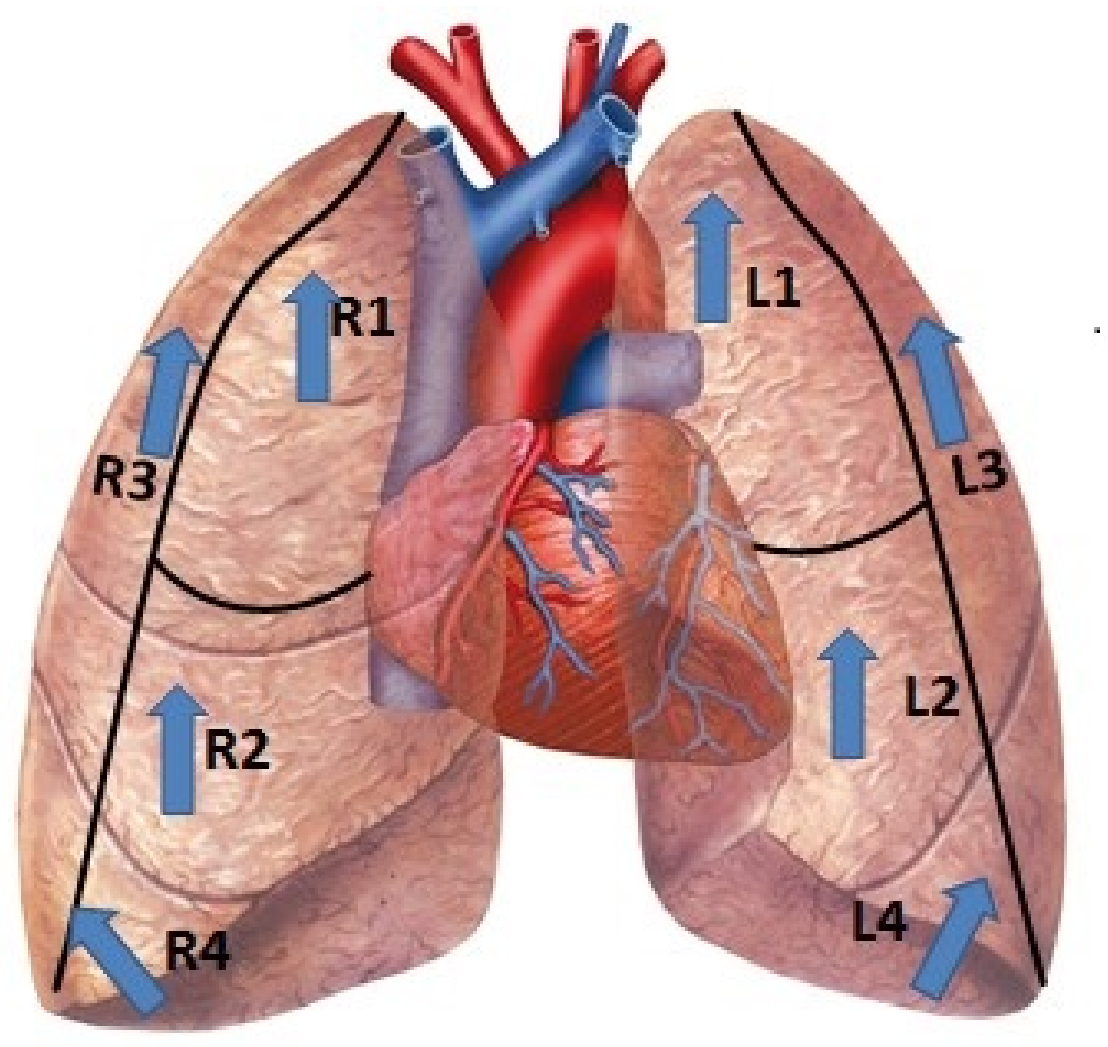




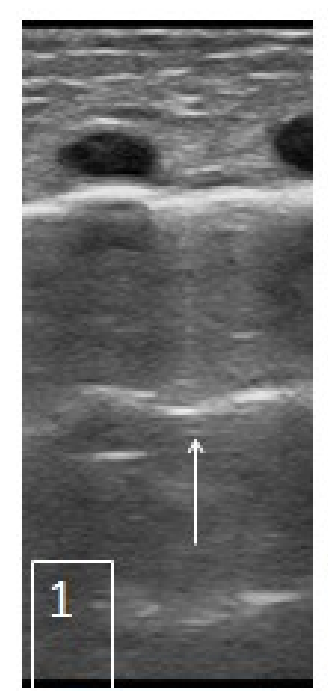

Score 0

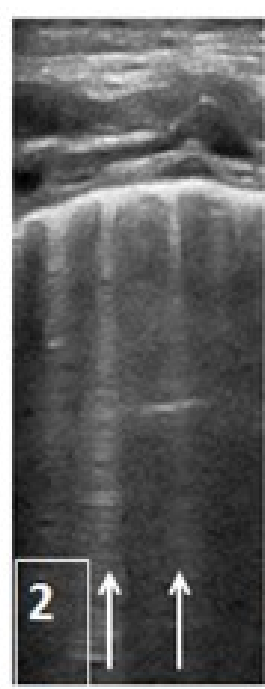

Score 1

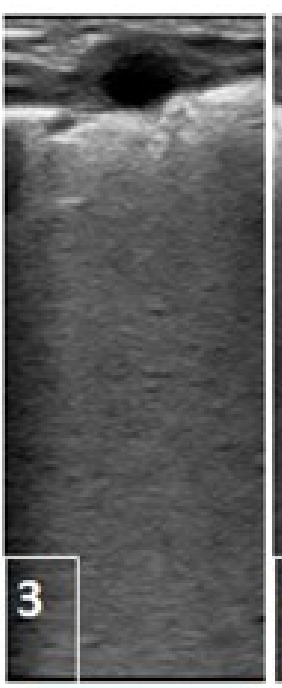

Score 2 Score 3
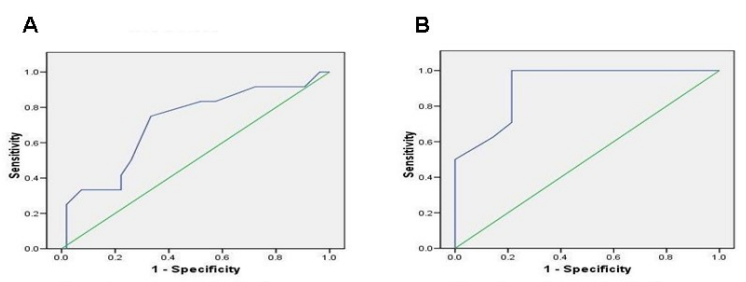

c

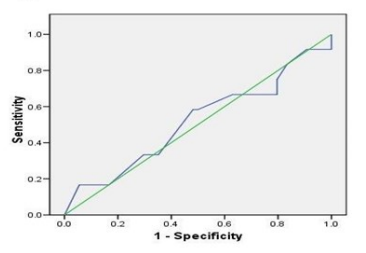

